\title{
MIDFOOT CHARCOT ARTHROPATHY IN DIABETIC PATIENTS: COMPLICATION OF AN EPIDEMIC DISEASE
}

Ricardo Cardenuto Ferreira', Daniel Hidalgo Gonçalez², João Manoel Fonseca Filho², Marco Túlio Costa ${ }^{3}$, Roberto Attilio Lima Santin ${ }^{4}$

\section{ABSTRACT}

Objectives: To outline the epidemiological profile of diabetic patients with Charcot arthropathy affecting the midfoot alone or extending from the midfoot to the hindfoot; To assess the results from the treatment that these patients undergo, according to a preestablished protocol, over the medium term. Methods: We retrospectively evaluated 88 patients (110 extremities) with Charcot arthropathy of the midfoot. The minimum follow-up period was 12 months. We included 45 patients with Charcot arthropathy affecting the tarsal-metatarsal joints $(51 \%) ; 20$ patients in whom the talonavicular, calcaneocuboid and subtalar joints were affected (23\%); and 23 patients in whom both the midfoot and hindfoot were affected (26\%), as described by Brodsky and Trepman. We defined the treatment as successful when a functional foot was preserved; and unsuccessful when the foot was amputated. Results: From treating Charcot arthropathy primarily involving the midfoot were satisfactory in the cases of 75 patients $(85 \%)$ treated according to our protocol. For the patients with severe lesions affecting both the midfoot and the hindfoot, a greater number of complex operations (i.e. arthrodesis) were needed in order to obtain the same overall rate of satisfactory results. The osteoarticular lesions originating in the midfoot probably extended progressively to the hindfoot because of delayed diagnosis with inadequate early treatment. Conclusion: It was possible to preserve a functional extremity in $85 \%$ of the patients. Severe lesions involving the midfoot and extending to the hindfoot required a greater number of surgical procedures to treat them.

Keywords - Arthropathy, Neurogenic; Diabetes; Foot; Amputation

\section{INTRODUCTION}

Diabetes is a chronic disease with high prevalence in modern society and is closely associated with obesity ${ }^{(1,2)}$. It constitutes a public health problem because it is now of epidemic proportions worldwide, with 171 million people affected in the year $2000^{(3)}$.

Introduction of insulin in treating the disease has provided improvements in blood glucose control and has increased patients' survival. Consequently, late-stage complications of diabetes that were only rarely observed in the past have become noted with greater frequency ${ }^{(4,5)}$.

Peripheral neuropathy is the main cause of late complications in the feet of diabetic patients. De- formities resulting from intrinsic muscle paralysis, loss of protective muscle paralysis and destruction of bones and joints of the foot and ankle are the three most common occurrences that predispose towards appearance of pressure ulcers on the feet ${ }^{(6)}$.

Charcot arthropathy, which affects the feet and ankles, is becoming a relatively common problem among diabetic patients, especially those in whom the disease has been developing for more than 10 years ${ }^{(1,7)}$.

Destruction and disarrangement of the osteoarticular architecture of the foot may create deformities and instabilities, with subsequent appearance of bone prominences, particularly when the lesion affects the midfoot and provokes collapse of the medial $\operatorname{arch}^{(8-11)}$.

\footnotetext{
1 - PhD. Professor and Head of the Foot and Ankle Surgery Group, Department of Orthopedics and Traumatology, Santa Casa de São Paulo, São Paulo, SP, Brazil.

2 - Third-year Resident Physician in Orthopedics and Traumatology, Santa Casa de São Paulo, São Paulo, SP, Brazil.

3 - MSc. Attending Physician in the Foot and Ankle Surgery Group, Department of Orthopedics and Traumatology, Santa Casa de São Paulo, São Paulo, SP, Brazil.

4 - PhD. Professor and Consultant in the Foot and Ankle Surgery Group, Department of Orthopedics and Traumatology, Santa Casa de São Paulo, São Paulo, SP, Brazil.

Work performed in the Department of Orthopedics and Traumatology, School of Medical Sciences, Santa Casa de São Paulo. Director: Prof. Dr. Osmar Avanzi.

Correspondence: Daniel Hidalgo Gonçalez, Rua Dr. Cesáreo Motta Júnior 112, 2176-7000 São Paulo, SP. E-mail: danielhidalgog@gmail.com
}

Work received for publication: September 26, 2011; accepted for publication: November 29, 2011.

The authors declare that there was no conflict of interest in conducting this work 
Early diagnosis of Charcot arthropathy and immediately starting treatment may be decisive in the evolution of the disease, with regard to preservation or amputation of the foot ${ }^{(6)}$.

The aims of the present study were: 1) to outline the epidemiological profile of diabetic patients with Charcot arthropathy exclusively affecting the midfoot or extending from the midfoot to the hindfoot; and 2) to evaluate, over the medium term, the result from the treatment that these patients undergo, in accordance with a preestablished protocol. In this study, we defined success as preservation of a functional foot and failure as amputation of the foot. Our hypothesis was that, in our setting, the diagnosis of Charcot arthropathy is made late, and that this delays the start of treatment and may contribute negatively towards the outcome. We believe in the hypothesis that systematization of orthopedic treatment may contribute towards reducing the frequency of amputations.

\section{SAMPLE AND METHODS}

\section{Epidemiological factors}

Over a 12-year period, from March 1998 to June 2011, 580 diabetic patients were treated at the specialized outpatient clinic for foot and ankle surgery of the Department of Orthopedics and Traumatology of our institution. Among these, 174 patients (30\%) (206 lower-limb extremities) presented Charcot arthropathy affecting the foot and ankle. For this study, we selected the diabetic patients with peripheral neuropathy, loss of protective sensitivity of the extremity and Charcot arthropathy that exclusively affected the Lisfranc joint (Brodsky type I) ${ }^{(12)}$, talonavicular, calcaneocuboid and subtalar joints (Brodsky type II) or midfoot joints with extension to the hindfoot (Trepman type IV $)^{(13)}$. In this series, we excluded patients whose minimum follow-up was less than 12 months and those whose medical files did not contain sufficient information for this study. In this manner, our final sample was formed by 88 patients, of whom 22 were affected bilaterally, thus totaling 110 extremities.

The patients were invited to come for a clinical reevaluation between March and December 2010. Twenty-one of these 88 patients $(24 \%)$ responded to the call. These patients were interviewed and underwent physical and radiographic examinations. The information on the other 67 patients $(76 \%)$ who could not come in person for reevaluation was gathered from the institution's medical files. These files contained their histories, data on clinical and laboratory tests, and photographs and radiographs of the affected extremities. The mean age of these 35 female patients and 53 male patients at the start of their outpatient follow-up was 59 years (range from 32 to 87 years). The mean length of follow-up was 21 months.

Type II diabetes was more prevalent, affecting 80 of the 88 patients $(91 \%)$. Fifty-five patients were making regular use of insulin $(62 \%)$. The mean time that had elapsed between the appearance of the first symptoms of diabetes and the start of Charcot arthropathy was 12 years (ranging from zero and 44 years). Among the eight patients (9\%) with type I diabetes, the mean interval between the diagnosing of the disease and the start of symptoms of Charcot arthropathy was 19 years, while in the group of patients with type II diabetes, this mean interval was 11 years.

Twenty-eight patients (32\%) were smokers and 24 (27\%) were drinkers. An association between these two habits was found in 16 patients (18\%). Smoking and drinking were defined in accordance with the criteria of the World Health Organization (WHO) ${ }^{(14,15)}$.

The presence of some degree of vascular disease, even without any apparent clinical repercussion, was detected in 41 of the 88 patients $(47 \%)$. The criteria for evaluating this disorder involved comparative palpation of the anterior and posterior distal tibial pulses between the two extremities and observation of the capillary filling time, which was considered to be normal when this took not more than three seconds.

At the time when these 88 patients arrived at our outpatient clinic, the anatomical location of the lesions was assessed based on radiographic images and presented the following distribution: tarsometatarsal joints (TM) (Brodsky type I) in 45 patients (51\%); talonavicular (TN), calcaneocuboid (CC) and subtalar (ST) joints (Brodsky type II) in 20 (23\%); and midfoot joints with extension to the hindfoot (Trepman type IV) in 23 patients (26\%) (Table 1).

The stage of evolution of the disease was also evaluated at the start of the outpatient follow-up, in accordance with the Eichenholtz classification ${ }^{(16)}$. The following distribution was observed among the 88 patients: in $41(47 \%)$, the lesion was at the initial phase of bone fragmentation (type I); in 17 (19\%), in the hypertrophic phase of consolidation (type II); and in $30(34 \%)$, in the late phase of sequelae (type III). 
Table 1 - Profile of the 88 patients in the study regarding the risk factors associated with type I and II diabetes. Source: SAME ISCMSP.

\begin{tabular}{c|c}
\hline Factor evaluated & Value \\
\hline Mean age & 59 years \\
\hline Bilaterality & $22(25 \%)$ \\
\hline Type I diabetes & $8(9 \%)$ \\
\hline Type II diabetes & $80(91 \%)$ \\
\hline $\begin{array}{c}\text { Mean time elapsed between diagnosing of diabetes } \\
\text { and appearance of Charcot arthroplasty }\end{array}$ & 12 anos \\
\hline Insulin use & $55(62 \%)$ \\
\hline Smoking & $28(32 \%)$ \\
\hline Drinking & $24(27 \%)$ \\
\hline Smoking and drinking & $16(18 \%)$ \\
\hline Vascular disease & $41(47 \%)$ \\
\hline
\end{tabular}

\section{Treatment protocol for Charcot arthropathy}

In our institution, the protocol for treating Charcot arthropathy of the midfoot is based and guided by the stage of evolution of the disease ${ }^{(16)}$. Active lesions in the phase of bone fragmentation (stage I) are characterized clinically by edema, hyperemia and hyperthermia of the foot and ankle. On simple radiographs of the affected foot, we observed osteopenia, periarticular fragmentation, subluxation or even joint dislocation (Figure 1). The patients were treated by means of total contact plaster casts (TCC) until the inflammatory phase had been resolved ${ }^{(17,18)}$. The transition from the fragmentation phase to the coalescence phase (stage II) was determined by the clinical observation of reductions of the edema, temperature and hyperemia. On radiographs, we checked for the presence of absorption, bone consolidation and bone sclerosis ${ }^{(12,19)}$ (Figure 2). For the patients who reached the coalescence phase, the TCC was replaced by an orthotic boot made of AFO polypropylene extending from mid-calf to the foot, together with use of protective footwear made-tomeasure for extra-deeply insensitive feet. This orthosis was then kept in use until complete bone consolidation had been achieved (Eichenholtz phase III), characterized by complete absence of inflammatory signs in physical examinations and by the presence of radiographic consolidation (bone callus and trabeculae crossing the fracture lines) (Figure 3).

When bone consolidation had been achieved, the patient was instructed to use protective footwear for extra-deeply insensitive feet. Made-to-measure insoles were prescribed with the aim of accommodating residual deformities resulting from skewed consolidation, especially in cases with a collapsed medial
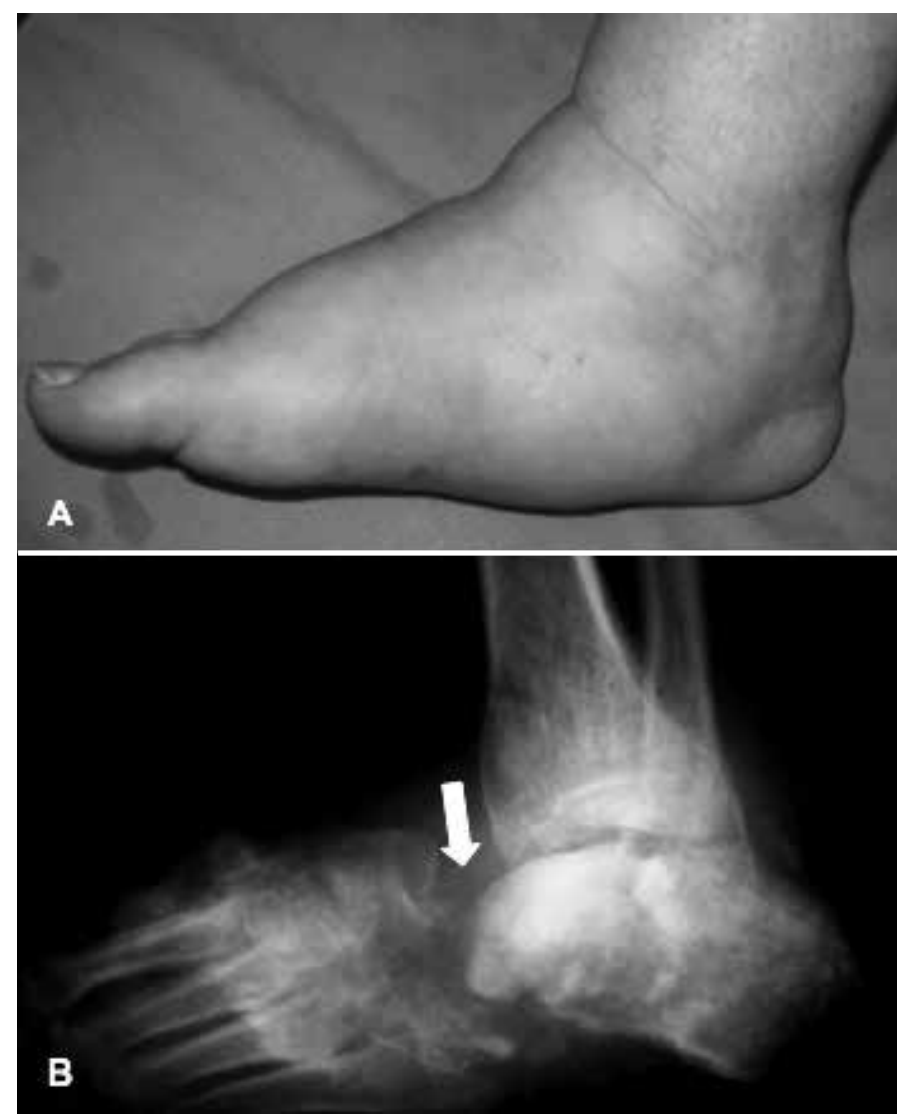

Figure 1 - Profile of the right foot showing diffuse edema and collapse of the medial plantar arch $(4 \mathrm{~A})$. Lateral radiograph of the foot $(4 \mathrm{~B})$ showing lesion affecting both the midfoot and the hindfoot (Trepman type IV). Diffuse osteopenia and multiple fractures-dislocations can be seen, involving the tarsal bones, which are typical lesions of Eichenholtz evolutional stage I. The arrow indicates joint dissociation at the level of the Chopart joint (TN and CC). Note also the collapse of the arch and the equinus position of the hindfoot bone (talus and calcaneus), shown in the dotted circle.

Source: SAME ISCMSP.

plantar arch or presence of plantar bone prominences, which are generally located in the midfoot region. If the foot or ankle was seen to present instability after the consolidation phase, we indicated associated use of an orthotic boot covering from mid-calf to the foot on a permanent basis.

We indicated surgery for removing plantar bone saliencies (exostectomy) when the foot was in a stable condition, with adequate circulation, but there may be plantar prominences located in the midfoot (Schon grades Band C) ${ }^{(10)}$ with concomitant formation of recurrent ulcers that are refractory to conservative treatment (debridement and TCC) ${ }^{(20)}$.

In cases in which the residual deformity is very accentuated, or when there is major residual deformity, extending secondarily to the hindfoot (Trepman grade IV) ${ }^{(13)}$, or it is impossible to fit the foot into a molded orthotic boot made of AFO polypropylene, reconstructive surgery (modeling arthrodesis) is indicated 

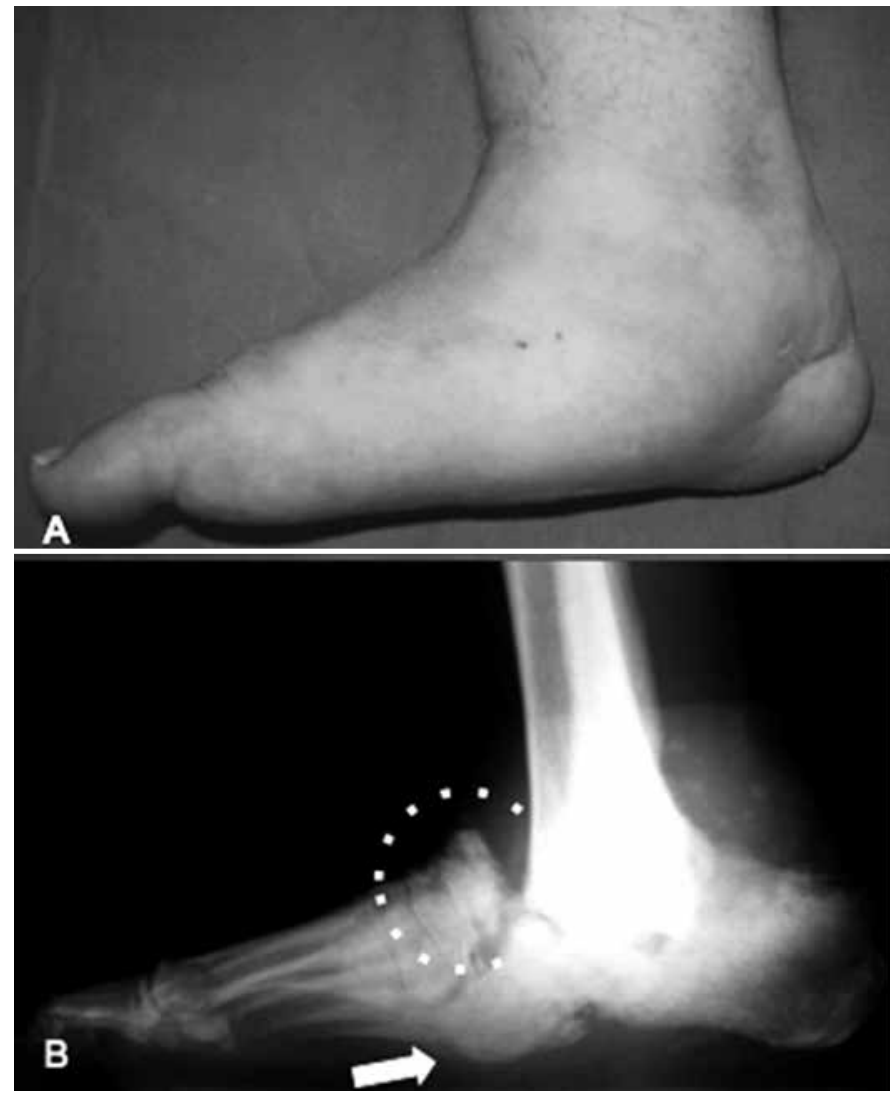

Figure 2 - Profile of the right foot of the same patient as in Figure 1, showing reduction of the edema following treatment with TCC (5A). The collapse of the medial plantar arch is more evident. Lateral radiograph of the foot (5B) showing extensive area of bone neoformation (dotted circle) typical of Eichenholtz evolutional stage II. Note the prominence of the head of the talus (arrow) in the plantar region of the midfoot, thus characterizing collapse of the medial arch and formation of a so-called blotter lesion.

Source: SAME ISCMSP.

in order to try to save the foot from possible amputation $^{(19)}$. After the reconstructive surgery, a prolonged period of immobilization with TCC is indicated, until bone consolidation or stable ankylosis of the foot or ankle is achieved.

Amputation of the foot may be indicated in the presence of severe infection of soft tissue or bone tissue, or in cases in which there is significant deformity with concomitant insufficient circulation of the feet, or after failure of surgical treatment ${ }^{(21-24)}$.

\section{Evaluation of the results after treatment}

To evaluate the results, we divided the treatment of Charcot arthropathy of the midfoot according to two possible types of outcome: 1) satisfactory, characterized by preservation of a functional foot capable of supporting the body's weight for independent walking, even if this required use of an orthosis of AFO type or a walking stick; 2) unsatisfactory, characterized by amputation
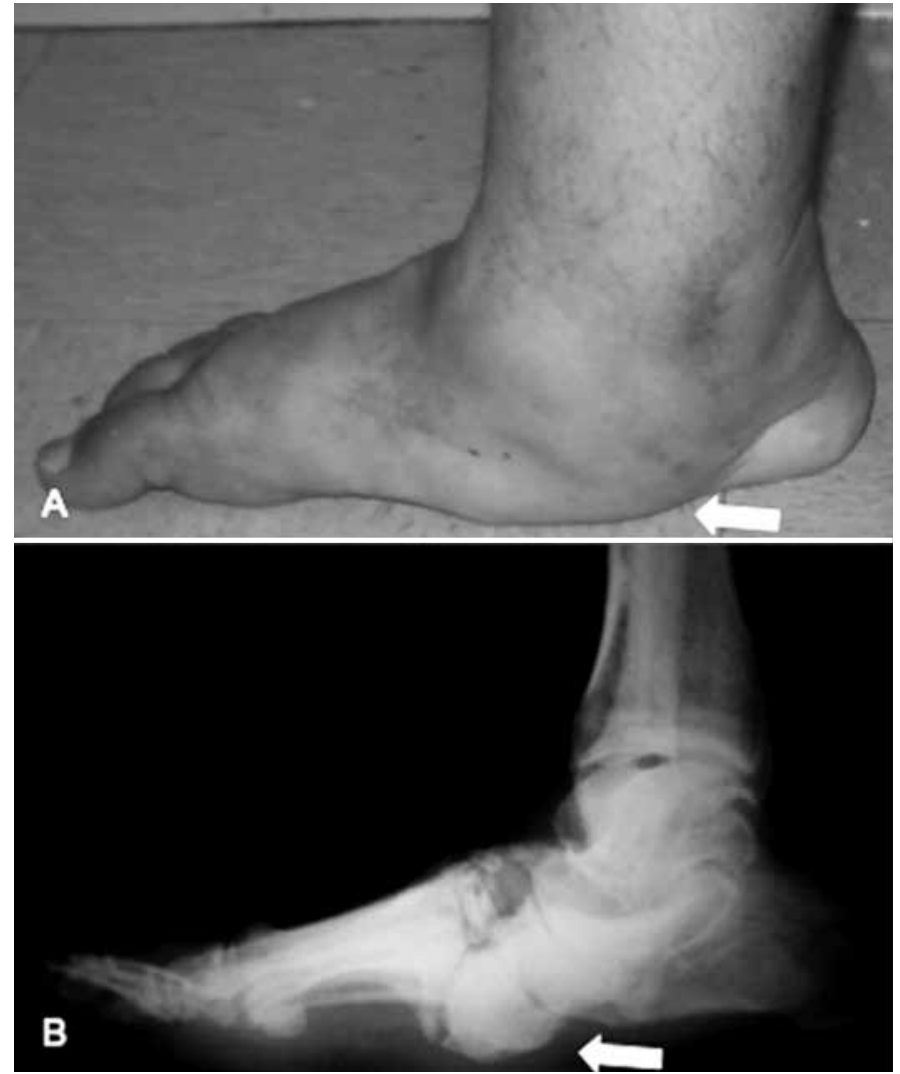

Figure 3-Profile of the right foot of the same patient as in Figures 1 and 2 after completing the treatment with TCC, showing the plantigrade position of the foot, now without detectable edema but with marked collapse of the medial arch (6A). Note also the prominence in the plantar region, characterizing the blotter appearance of the foot (arrow). Lateral radiograph of the foot (6B) with signs of bone consolidation, characteristic of Eichenholtz evolutional stage III. Note the persistence of a large bone prominence present in the plantar region of the midfoot (arrow), which is an area at risk of ulceration. Source: SAME ISCMSP.

consequent to complications from Charcot arthropathy or by maintenance of a foot that was incapable of supporting the body's weight while walking (with use of crutches or a wheelchair).

Lesser amputations involving only the forefoot (of the toes or rays, or transmetatarsal or at the level of the Lisfranc joint) were not considered to be relevant in the final evaluation, unless they were directly related to Charcot arthropathy with the midfoot affected. This criterion was used when the triggering factor for amputation of the forefoot was the presence of infected or ischemic ulcers.

Deaths caused by alterations relating to Charcot arthropathy were considered to be an unsatisfactory result from the treatment.

Risk factors such as bilaterality, smoking, drinking, interval between onset of diabetes and appearance of Charcot arthropathy, insulin use, vascular disorders, sensory disorders, stage of evolution and initial loca- 
tion affected by Charcot arthropathy were correlated in order to try to assess the possible influence of these factors on the result from treating the disease.

For the statistical analysis, the SPSS 13.0 software for Windows was used. Descriptive measurements of the quantitative variables and the absolute and relative frequencies of the qualitative variables were obtained. The chi-square or Fisher exact test was used to compare qualitative variables and Student's t test was used to compare qualitative variables with quantitative variables.

The possible costs resulting from surgical treatment were inferred indirectly by taking into consideration the number of operations performed on patients in a severe condition.

\section{RESULTS}

Our sample was formed by 88 patients, of whom 22 was affected bilaterally, thus totaling 110 extremities. The mean age of the 35 female patients and 53 male patients at the start of the outpatient follow-up was 59 years (ranging from 32 to 87 years) and the mean length of follow-up was 21 months (ranging from 13 to 73 months).

At the time when the 88 patients arrived at our outpatient clinic, the anatomical location of the lesions presented the following distribution: TM joints (Brodsky type I) in 45 patients $(51 \%) ; \mathrm{TN}, \mathrm{CC}$ and ST joints (Brodsky type II) in 20 (23\%); and midfoot joints with extension to the hindfoot (Trepman type IV) in 23 patients $(26 \%)$.

The stage of evolution of the disease at the time of the first consultation had the following distribution: in 41 patients $(47 \%)$, the lesion was in the initial phase of bone fragmentation (Eichenholtz type I); in 17 $(19 \%)$, it was in the consolidation phase (Eichenholtz type II); and in 30 (34\%), it was in the late phase of sequelae (Eichenholtz type III).

Through correlating the anatomical location with the stage of evolution of the Charcot arthropathy, we observed that out of the 45 patients with the TM joint affected (Brodsky I), 22 (49\%) presented the disease in the evolutional phase of fragmentation (Eichenholtz type I); six (13\%) were in the consolidation phase (Eichenholtz type II); and 17 (38\%) were in the phase of sequelae (Eichenholtz type III) (Figure 4). In this group of 45 patients with the TM joint affected, closed treatment was performed suc-

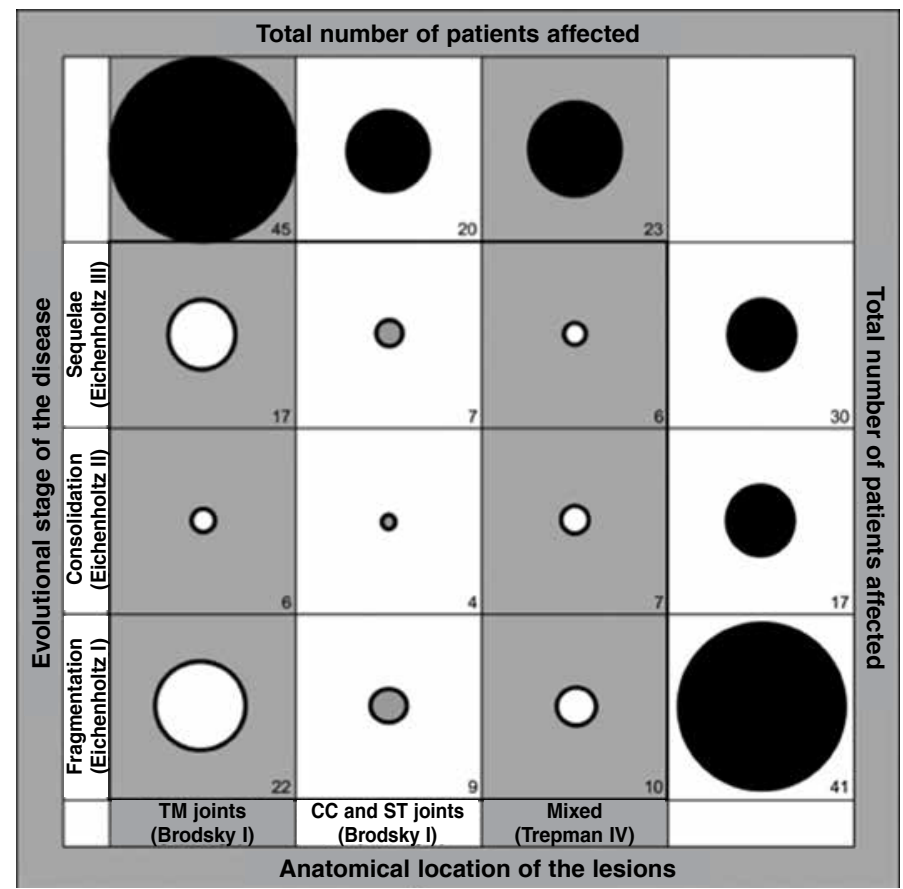

Figure 4-Distribution of the 88 patients with Charcot arthroplasty in relation to the anatomical location of the lesions and the evolutional stage of the disease. The numerical values represent the numbers of patients; the size of the circles is proportional to the number of patients affected.

TM: tarsometatarsal; TN: talonavicular; CC: calcaneocuboid; ST: subtalar. Source: SAME ISCMSP.

cessfully in 35 (78\%). Some type of surgery was required for 10 patients $(22 \%)$, distributed thus: plantar exostectomy in four patients; modeling arthrodesis in another four; and amputation of the extremity in another two (Figures 5 and 6).

Among the 20 patients who presented Charcot arthropathy affecting the TN, CC and ST joints (Brodsky type II), nine (45\%) had the disease in the evolutional phase of fragmentation (Eichenholtz type I); four $(20 \%)$ were in the evolutional phase of consolidation (Eichenholtz type II); and seven (35\%) were in the phase of sequelae (Eichenholtz type III) (Figure 4). In this group of 20 patients with the TN, CC and ST joints affected, closed treatment was successfully performed in $12(60 \%)$. Some type of surgery was required for eight patients (40\%), distributed thus: plantar exostectomy in four patients; modeling arthrodesis in another three; and amputation of the extremity in a single patient (Figures 5 and 6).

Among the 23 patients who presented Charcot arthropathy involving the midfoot and extending to the hindfoot (Trepman type IV), ten (43\%) had the disease in the evolutional phase of fragmentation (Eichenholtz type I); seven (30\%) were in the evolutional phase of consolidation (Eichenholtz type II); and six $(26 \%)$ were in the phase of sequelae (Eichenholtz 


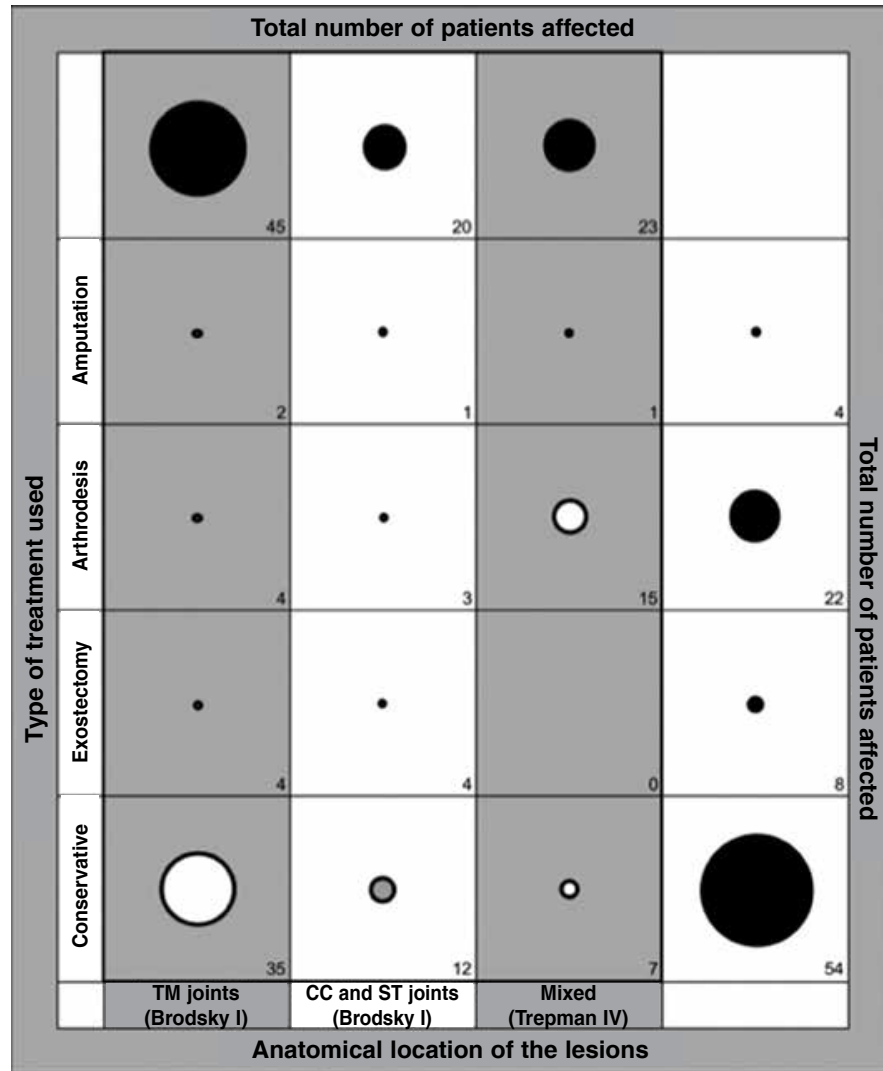

Figure 5 - Distribution of the 88 patients with Charcot arthroplasty in relation to the anatomical location of the lesions and the type of treatment used. The numerical values represent the numbers of patients; the size of the circles is proportional to the number of patients affected.

TM: tarsometatarsal; TN: talonavicular; CC: calcaneocuboid; ST: subtalar. Source: SAME ISCMSP.

type III) (Figure 4). In this group of 23 patients with the midfoot affected and extension to the hindfoot, conservative treatment was successfully performed in seven $(30 \%)$. Some type of surgery was required for 16 patients $(70 \%)$, distributed thus: modeling arthrodesis in 15 patients and amputation of the extremity in a single patient (Figures 5 and 6).

Analysis on seven risk factors (1 - age; 2 - type of diabetes; 3 -insulin use; 4 - bilaterality; 5 - smoking; 6 - drinking; and 7 - vascular diseases) that have all been associated with higher incidence of complications in treating Charcot arthropathy in diabetic patients, we found that among 75 of the 88 patients $(85 \%)$, whose results from the treatment were considered satisfactory, the mean age was 59 years; 68 of these 75 patients $(91 \%)$ presented type II diabetes; $47(63 \%)$ were using insulin to clinically control the disease; 17 (23\%) were affected bilaterally; 22 (29\%) were smokers; 19 (25\%) were drinkers; and 36 (48\%) presented clinical signs of vascular disease. Among the group of 13 of the 88 patients (15\%), whose outcome was considered unsatisfactory, the mean age

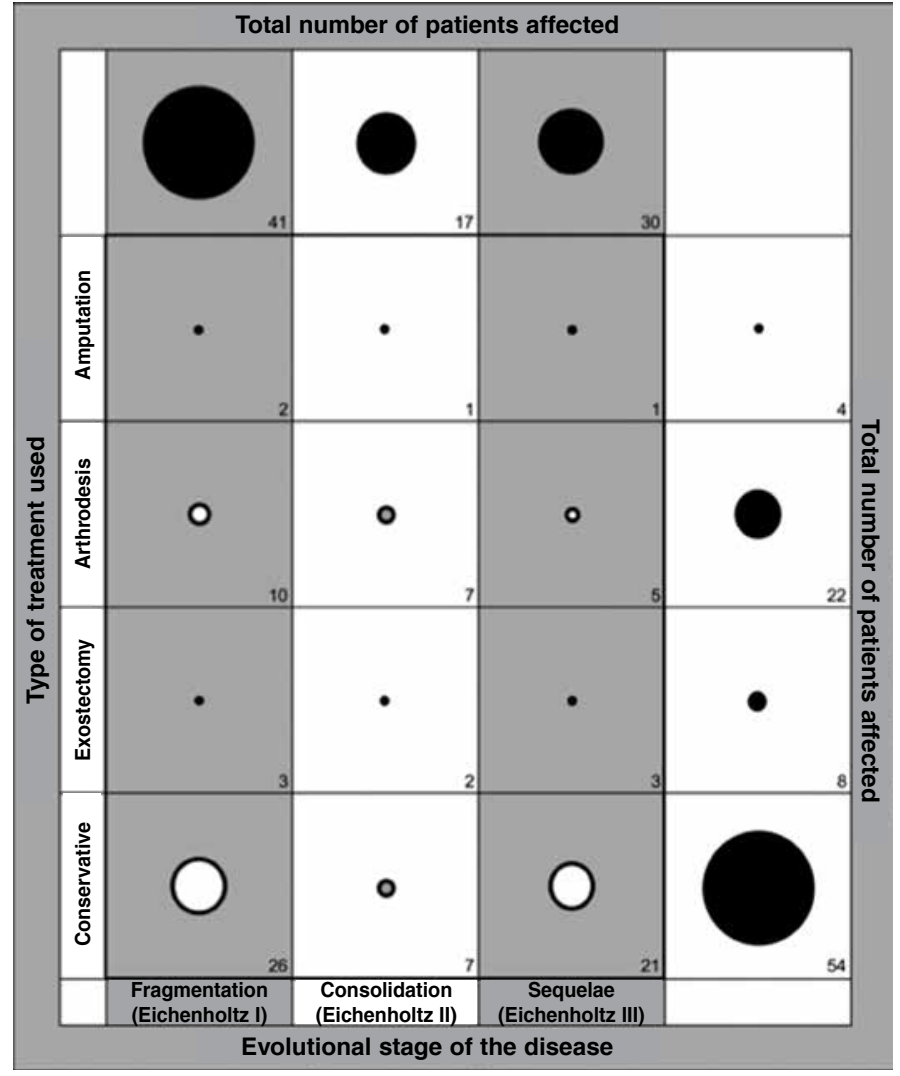

Figure 6 - Distribution of the 88 patients with Charcot arthroplasty in relation to the evolutional stage of the disease and the type of treatment used. The numerical values represent the numbers of patients; the size of the circles is proportional to the number of patients affected.

Source: SAME ISCMSP.

was 62 years; 12 of these 13 patients $(92 \%)$ presented type II diabetes; seven (63\%) were using insulin to clinically control the disease; five (38\%) were affected bilaterally; six (29\%) were smokers; five (38\%) were drinkers; and five (38\%) presented clinical signs of vascular disease. The sample size was insufficient for us to identify statistical significance in all the associated factors, but we observed a tendency towards unsatisfactory results among the patients bilaterally affected by Charcot arthropathy who were smokers or drinkers.

In correlating the anatomical locations of the lesions (types I, II or IV) with the outcome from the treatment (satisfactory or unsatisfactory), we found that among the 75 patients whose results were considered satisfactory, the lesions had the following distribution: TM joints (Brodsky type I) in 39 patients (52\%); TN, CC and ST joints (Brodsky type II) in 17 (23\%); and lesions of mixed type, extending from the midfoot to the hindfoot (type IV) in $19(25 \%)$. In the group of 13 patients whose results from the treatment were considered unsatisfactory, the anatomical 
distribution of the lesions in the extremities was as follows: TM joint (Brodsky type I) in six patients (46\%); TN, CC and ST joints (Brodsky type II) in three $(23 \%)$; and midfoot affected primarily with extension to the hindfoot (Trepman type IV) in four (31\%). No significant difference was seen through correlating the anatomical distribution of the lesions with the outcome from the treatment (satisfactory or unsatisfactory).

From correlating the evolutional stage of Charcot arthropathy (as classified by Eichenholtz) with the final outcome from the treatment (satisfactory or unsatisfactory), we found that in the group of 75 patients whose final result was considered to be satisfactory, $35(47 \%)$ were in the initial phase of fragmentation (Eichenholtz stage I); 14 (19\%) were in the bone consolidation phase (Eichenholtz stage II); and 26 $(35 \%)$ were in the phase of sequelae (Eichenholtz stage III). In the group of 13 patients whose final result was considered unsatisfactory, six (46\%) were in stage I; three (23\%) in stage II; and four $(31 \%)$ in stage III. There was no significant difference in correlating the distribution of the evolutional stage of Charcot arthropathy with the outcome from the treatment (satisfactory or unsatisfactory.

In correlating the results with the type of treatment used, we found that closed treatment, which was used for 54 of the 88 patients $(61 \%)$ of our sample, presented results that were considered satisfactory in 49 cases (91\%) and unsatisfactory in five $(9 \%)$. In relation to surgical treatment, which was performed for 34 of the 88 patients $(39 \%)$, the outcome was satisfactory in 26 cases $(76 \%)$ and unsatisfactory in eight (24\%). We observed a tendency towards satisfactory results among the patients who received closed treatment, compared with those who required some type of surgery, but it was not possible to identify statistical significance because the sample size was insufficient.

In comparing the type of surgery performed with the final result, we observed that all of the eight patients who only underwent exostectomy of a plantar bone prominence presented satisfactory results. On the other hand, among the 23 patients who underwent modeling arthrodesis, satisfactory results were achieved in 18 (78\%). One of the five patients whose results from modeling arthrodesis were considered unsatisfactory required amputation of the extremity; two patients remained with the affected limb deformed, unstable and incapable of bearing body weight or fitting with an orthosis; and the other two patients died due to complications such as postoperative infection. For three patients, amputation of the extremity was indicated primarily as the form of treatment.

In subdividing the 88 patients of our sample according to the extent of Chart arthropathy, we established two distinct groups: group I - lesion located exclusively in the midfoot (Brodsky types I and II), in 65 patients (74\%); and group II - lesion extending from the midfoot to the hindfoot (Trepman type IV), in 23 patients $(26 \%)$. Comparing the treatment outcomes between these two groups, we did not find any statistically significant difference with regard to satisfactory results, which were achieved in the cases of 54 of the 65 patients $(86 \%)$ in whom the lesion was restricted to the midfoot (group I) and in 19 of the 23 patients $(83 \%)$ in whom the lesions affected both the midfoot and the hindfoot (group II). However, regarding the need for surgery during the course of the treatment, we observed that in group I, only 18 of the 65 patients $(28 \%)$ needed to be operated, whereas in group II surgery was needed for 16 of the 23 patients $(70 \%)$. This difference was statistically significant ( $\mathrm{p}<0.001)$, thus demonstrating that there was a need for surgery during the treatment among the patients whose lesions extended from the midfoot to the hindfoot, for a satisfactory result to be obtained.

In analyzing the type of surgery needed during the course of the treatment, in the group of 65 patients in group I (affected exclusively in the midfoot), primary amputation was indicated in two patients; exostectomy of a plantar bone prominence was successfully performed in eight patients; and modeling arthrodesis of the extremity was necessary in another eight patients, with success in six cases. In the two patients who underwent modeling arthrodesis whose outcome was unsatisfactory, one developed an unstable deformity of the extremity and the other died due to complications relating to postoperative infection. Out of the total of 18 patients who were exclusively affected in the midfoot and underwent surgical treatment, the outcome was considered to be satisfactory in $14(78 \%)$.

In the group of 23 patients who were affected in the midfoot with extension to the hindfoot (group II), 
surgical treatment was necessary in $16(70 \%)$. Primary amputation of the extremity was indicated for one patient and modeling arthrodesis for the other 15. The final result was considered satisfactory in the cases of 12 of these 16 patients (75\%). Among the three patients who underwent modeling arthrodesis and had unsatisfactory outcomes, one developed unstable deformity of the extremity and the other two died due to complications relating to postoperative infection. In comparing the patients with lesions restricted to the midfoot (group I) with those whose lesions extended from the midfoot to the hindfoot (group II), we did not observe any statistically significant difference in relation to the result from the treatment. However, to attain the same number of satisfactory results as obtained in group I, a significantly greater number of surgical procedures were needed among the patients whose midfoot and hindfoot were both affected (group II).

\section{DISCUSSION}

The sample of 88 patients (110 extremities) in the present study was derived from the register of all of the 580 patients who were being followed up at our specialized outpatient clinic and were treated for foot conditions relating to late-stage complications from diabetes. Because our clinic is at a university hospital that provides tertiary-level orthopedic care and is a referral center for a large part of the city of São Paulo, we attend large numbers of previously screened patients presenting severe complications. The diagnosis of Charcot arthropathy was confirmed by means of radiographs on the feet of 174 patients, i.e. approximately $30 \%$ of all the diabetic patients followed up at out outpatient clinic. Of these, 88 patients fulfilled the selection and inclusion criteria for this study, thus corresponding to approximately $51 \%$ of all of the cases of Charcot arthropathy identified at out clinic and $15 \%$ of all the patients with late complications from diabetes in their feet.

The Department of Orthopedics and Traumatology of the Irmandade da Santa Casa de Misericórdia de São Paulo is located in the central region of Greater Grande São Paulo, an area with 18 million inhabitants $^{(25)}$, and it attends patients who mostly do not have health insurance. Thus, it can be considered that our sample depicted a relatively significant portion of the diabetic patients who sought the public healthca- re system for treatment of severe problems affecting their feet.

In analyzing the characteristics of the diabetic patients with Charcot arthropathy that primarily affected the midfoot and those whose disease also extended to the hindfoot, we identified the following patterns: elderly patients whose mean age was around 59 years; long duration of the disease, with a mean reaching 12 years; predominance of type II disease (in $91 \%$ of the cases); and high number of insulin-dependent patients $(62 \%)$. These data, when added to the socioeconomic profile of the patients attended at our institution (low educational level and low income), give rise to special concern regarding the financial impact on public health. Charcot arthroplasty is one of the severe late-stage manifestations of diabetes and, in conjunction with other complications such as nephropathy, retinopathy and cardiocirculatory disorders ${ }^{(2)}$, it has the potential to cause high expenditure, thus consuming enormous amounts of financial resources destined for public health.

In our sample, $32 \%$ of the patients were smokers and $27 \%$ were drinkers. These are vices that have been widely recognized to have an unfavorable impact when associated with diabetes ${ }^{(26)}$. We believe that the misinformation that is typically correlated with patients who smoke and drink can be combated through educational programs.

Some degree of peripheral vascular disease was identified in $47 \%$ of the patients in our series. In the great majority of the cases, there was no evident clinical repercussion, given that our service receives patients who were previously screened and predominantly present orthopedic conditions.

In our series, $22(25 \%)$ of the 88 patients presented bilateral occurrence of Charcot arthropathy. The treatments for these patients resulted in unfavorable outcomes in five cases $(23 \%)$, thus showing a tendency towards worse results than among the patients with unilateral lesions. In the literature, it has been shown that the results from treatments among patients affected bilaterally by Charcot arthropathy are worse than the outcomes in unilateral cases ${ }^{(16,27,28)}$. However, in our series, this could not be demonstrated due to the insufficient sample size.

Use of a systematized protocol for administering treatment for Charcot arthropathy affecting the midfoot involves minimizing the damage and reducing 
the risks of complications. Deformities, foot and ankle joint instability and plantar bone prominences are often associated with elevated pressure during the weight-bearing phase of gait. As a consequence, ulcers and secondary infections tend to arise, which may put the patient's extremity fatally at risk of amputation.

In our series, we found that the hindfoot and ankle were affected secondarily from the typical lesions of Charcot arthropathy that began in the midfoot. We believe that slowness in identifying osteoarticular lesions, both in TM joints (Brodsky type I) and in TN, CC and ST joints (Brodsky type II), especially delayed the start of treatment with TCC. Mistaken and late diagnosis were extremely common among the patients who sought attendance at our outpatient clinic, and the great majority of them reported that there had been a long interval between the appearance of the first symptoms and the definitive diagnosing of Charcot arthropathy. Because of diagnostic failures and late starts to treatment, we found that $26 \%$ of the patients in our series began outpatient treatment at an advanced stage of the disease, with lesion locations that had already extended from the midfoot to the hindfoot (Trepman type IV). We attribute this progression of the disease to the prolonged period during which the patients continued to bear weight on the lower extremity without any type of protection. This certainly contributed towards increasing the osteoarticular destruction and towards progression of the deformities, thus worsening the clinical condition and making the treatment more difficult.

In assessing the efficacy of the treatment protocol for Chart arthropathy affecting the midpoint that was used in this study, we noted that after a mean length of follow-up of 21 months, 75 of the 88 patients (85\%) presented satisfactory results, in which it was possible to achieve a plantigrade foot that was stable or could be accommodated in a molded orthosis of AFO type, with weight-bearing during gait. The anatomical location of the lesion (restricted to the midfoot or extending to the hindfoot), the evolutional stage of the disease at the time when the patient arrived at the outpatient clinic (Eichenholtz stages I, II or III) and the type of treatment needed (closed or surgical) did not influence the result from the treatment (satisfactory or unsatisfactory). However, we found that a significantly greater proportion $(70 \%)$ of the patients whose osteoarticular lesions extended from the midfoot to the hindfoot (Trepman type IV) required surgery than among the patients whose Charcot arthropathy was restricted to the midfoot (28\%).

Surgical indication for treating the problems relating to Charcot arthropathy was not optional among the criteria used in our protocol. Selection of the patients who were candidates for surgery followed the order of severity of the lesions. Plantar exostectomy was the simplest procedure, and was indicated for treating the bone prominences that were responsible for recurrent ulceration ${ }^{(19)}$. All the eight patients in our series who underwent this type of procedure had favorable evolution. Among the patients with severe unstable deformities affecting the midfoot or hindfoot who were incapable of plantigrade weight-bearing and were in imminent danger of developing pressure ulcers, or already presented recurrent ulceration, modeling arthrodesis was indicated with the objective of avoiding possible amputation of the extremity ${ }^{(21-24)}$. In our sample, modeling arthrodesis was successful in 18 of the 23 patients, thus attaining a salvage rate of $78 \%$ of the extremities operated. Two of the patients whose results were unsatisfactory after the salvage attempt using modeling arthrodesis died due to complications such as postoperative infection. In another two patients, both the deformity and the instability of the extremity persisted, thus impeding weight-bearing on the limb during gait; however, these patients refused to undergo amputation.

The worldwide incidence of diabetes is expected to increase over the coming years, especially in emerging countries ${ }^{(3)}$. In addition, improved clinical control over the disease has promoted greater life expectancy among diabetic patients, thus prolonging the period of exposure to the risks of late complications from the disease ${ }^{(4,5)}$. In this light, public healthcare bodies need to prioritize attention to basic education among the patients; to special programs for prevention and treatment of diabetes and its late-stage complications; to the infrastructure and preparedness of healthcare units; and to early treatment for lesions in feet caused by Charcot arthropathy. In this manner, effective directing of resources might avoid waste due to complex, prolonged and burdensome treatments that very often have poor results. 


\section{CONCLUSIONS}

The epidemiological profile of diabetic patients with Charcot arthropathy affecting the midfoot is characterized by manifestation of the disease in the third age, presence of type II diabetes and chronic insulin use, with osteoarticular lesions originating in the midfoot and progressively extending to the hindfoot, due to delayed diagnosis and a late start for appropriate treatment.

\section{REFERENCES}

1. Armstrong DG, Peters EJ. Charcot's arthropathy of the foot. J Am Podiatr Med Assoc. 2002;92(7):390-4.

2. Gilliland B. Neuropathic joint disease in relapsing polychonditis and other arthritis. In: Fauci A, Braunwald E, Isselbacher K, editors. Harrison's principles of internal medicine. 14th ed. 1998. New York: McGraw-Hill; 1953.

3. Wilde S, Roglic G, Green A, Sicree R, King H. Global prevalence of diabetes. Estimates for the year 2000 and projections for 2030. Diabetes Care. 2004;27(5):1047-53.

4. Bridges RM Jr, Deitch EA. Diabetic foot infections. Pathophysiology and treatment. Surg Clin North Am. 1994;74(3):537-55.

5. Pakarinen TK, Laine HJ, Honkonen SE, Peltonen J, Oksala H, Lahtela J. Charcot arthropathy of the diabetic foot. Current concepts and review of 36 cases. Scand J Surg. 2002;91(2):195-201.

6. Ferreira RC, da Silva APS, Costa MT, Frizzo GG, Santin RA Lima, Fucs PMM Aspectos epidemiológicos das lesões no pé e tornozelo do paciente diabético. Acta Ortop Bras. 2010;18(3):135-41.

7. Petrova N, Foster A, Edmunds M. Difference in presentation of Charcot osteoarthropathy in Type 1 compared with Type 2 Diabetes. Diabetes Care. 2004;27(5):1235.

8. Eloesser L. On the nature of neuropathic affections of the joints. Ann Surg. 1917;66:201-7

9. Salo PT, Theriault E, Wiley RG. Selective ablation of rat knee joint innervation with injected immunotoxin: a potential new model for the study of neuropathic arthritis. J Orthop Res. 1997;15(4):622-8.

10. Schon LC, Weinfeld SB, Horton GA, Resch S. Radiographic and clinical classification of acquired midtarsus deformities. Foot Ankle Int. 1998;19(6):394-404.

11. Schon LC, Easley ME, Weinfeld SB. Charcot neuroarthropathy of the foot and ankle. Clin Orthop Relat Res. 1998;(349):116-31

12. Brodsky JW. The diabetic foot. In: Coughlin MJ, Mann RA, Saltzman CL, editors: Surgery of the foot and ankle. 8th. ed. St. Louis: Mosby; 2006. p. 1281-368.

13. Trepman E, Nihal A, Pinzur MS. Current topics review: Charcot neuroarthropathy of the foot and ankle. Foot Ankle Int. 2005;26(1):46-63.

14. Organização Mundial de Saúde. Global Status Report on Alcohol 2004 Geneva:WHO;2004. [acesso em 23 abr 2011]. Disponível em: http://www. who.int/subtance_abuse/publications/globalstatusreportalcohol2004_ drinkpatterns.pdf.
In the great majority of our cases $(85 \%)$, the result from our treatment protocol for Charcot arthropathy affecting the midfoot was satisfactory. However, among the patients whose lesion extended from the midfoot to the hindfoot (type IV), the number of operations needed to attempt to preserve the extremity was significantly greater than among the patients whose Charcot arthropathy was restricted to the midfoot (types I and II).
15. Organização Mundial de Saúde. International statistical classification of diseases and related health problems. 10th ed. Geneva; 1992.

16. Hartemann-Heurtier A, Van GH, Grimaldi A. The Charcot foot. Lancet. 2002;360(9347):1776-9.

17. Pinzur MS, Lio T, Posner M. Treatment of Eichenholtz stage I Charcot foot arthropathy with a weightbearing total contact cast. Foot Ankle Int. 2006;27(5):324-9.

18. Adler AI, Boyko EJ, Ahroni JH, Smith DG. Lower-extremity amputation indiabetes. The independent effects of peripheral vascular disease, sensory neuropathy, and foot ulcers. Diabetes Care. 1999;22(7):1029-35.

19. Sanders LJ, Frykberg RG. The Charcot foot. In Bowker JH, Pfeifer MA, editors. Levin and O'Neal's the diabetic foot. 7th ed. Philadelphia: Mosby Elsevier; 2007. p. 258.

20. van der Ven $\mathrm{A}$, Chapman $\mathrm{CB}$, Bowker JH. Charcot neuroarthropathy of the foot and ankle. J Am Acad Orthop Surg. 2009;17(9):562-71.

21. Papa J, Myerson M, Girard P. Salvage, with arthrodesis, in intractable diabetic neuropathic arthropathy of the foot and ankle. J Bone Joint Surg Am. 1993;75(7):1056-66.

22. Stone NC, Daniels TR. Midfoot and hindfoot arthrodeses in diabetic Charcot arthropathy. Can J Surg. 2000;43(6):449-55.

23. Cooper PS. Application of external fixators for management of Charcot deformities of the foot and ankle. Foot Ankle Clin. 2002;7(1):207-54

24. Caravaggi C, Cimmino M, Caruso S, Dalla Noce S. Intramedullary compressive nail fixation for the treatment of severe Charcot deformity of the ankle and rear foot. J Foot Ankle Surg. 2006;45(1):20-4.

25. Instituto Brasileiro de Geografia e Estatística. [acesso em 17 de julho 2011]. Disponivel em: http://www.ibge.gov.br/cidadesat/link.php?uf=sp

26. Abbott CA, Carrington AL, Ashe H, Bath S, Every LC, Griffiths J, et al. The North-West Diabetes Foot Care Study: incidence of, and risk factors for, new diabetic foot ulceration in a community-based patient cohort. Diabet Med. 2002;19(5):377-84.

27. Sanders LF. Charcot neuroathropathy of the foot. In: Bowker J, Pfeifer M, editors. The diabetic foot. 6th edition. St. Louis: Mosby; 2001. p. 439-66.

28. Clohisy DR, Thompson RC Jr. Fractures associated with neuropathic arthropathy in adults who have juvenile-onset diabetes. J Bone Joint Surg Am. 1988;70(8):1192-200. 